\title{
Thermal Pyrolysis Characteristics and Kinetic Analysis of Nanofibrillated Cellulose/Graphene Oxide Composites
}

\author{
Xia Meng, ${ }^{\mathrm{a}}$ Shengdan Wang, ${ }^{\mathrm{a}}$ Wenhua Gao, ${ }^{\mathrm{b}} *$ Wenjia Han, ${ }^{\mathrm{a}}$ and Lucian A. Lucia ${ }^{\mathrm{c}}$ \\ The fabrication process determines the microstructure and physical \\ properties of material to some extent. In this study nanofibrillated cellulose \\ /graphene oxide (NFC/GO) composites were fabricated by casting and \\ evaporation methods, respectively. The microstructure and thermostability \\ of the composites were investigated. The composites fabricated by the \\ casting method had more uniform layered microstructure than those made \\ by the evaporation method. The thermogravimetry analysis indicated that \\ in the stage of 100 to $220^{\circ} \mathrm{C}$ diffusion, the mechanisms of the Ginstling- \\ Brounstein equation (D4) and the one-way transport equation (D1) can be \\ regarded as the most suitable thermal pyrolysis reaction models for the \\ composites made by the casting method and the evaporation method, \\ respectively. However, in the stage of 220 to $380^{\circ} \mathrm{C}$, the two types of \\ composites showed totally different thermal pyrolysis mechanisms. The \\ results manifested that forming methods could indeed affect the \\ microstructure and thermal degradation process of composites.
}

Keywords: Cellulose nanofibril; Thermal pyrolysis mechanism; Fabrication method

Contact information: a: State Key Laboratory of Biobased Material and Green Papermaking, Qilu University of Technology, Shandong Academy of Sciences, Jinan 250353, China; b: State Key Laboratory of Pulp and Paper Engineering, South China University of Technology, Guangzhou 510640, China; c: North Carolina State University, Departments of Chemistry, Forest Biomaterials, Raleigh, NC 27695, USA; *Corresponding author: segaowenhua@scut.edu.cn

\section{INTRODUCTION}

Nanofibrillated cellulose (NFC) is a new class of bio-products in the bio-refinery process and has received increased attention due to its potential value-added applications in the fields of biomaterial, biosensors, supercapacitors, pharmaceuticals, food, papermaking, and so on (Alemdar and Sain 2008; Long et al. 2017). Generally, NFC can be separated from lignocellulosic biomass, which consists of three main components: cellulose (38 to 50\%), hemicellulose (23 to 32\%), and lignin (15 to 25\%) (Sun et al. 2016). Enzymatic pretreatment, oxidation pretreatment, or alkaline pretreatment followed by mechanical treatment is the main fundamental procedure for NFC preparation (Xiang et al. 2016). The method of enzymatic pretreatment with high-pressure homogenization is considered an environmentally friendly method to produce NFC, due to the mild treatment conditions, significantly reduced energy consumption, and almost no chemicals used in the process (Beltramino et al. 2015; Wang et al. 2018). Various enzymes have been explored to facilitate NFC disintegration from lignocellulosic fibers, and it has been found that endoglucanase pretreatment could effectively promote disintegration of fibers to NFC (Penttila et al. 2013; Liu et al. 2016; Kanmani et al. 2017). Moreover, the NFC produced from enzymatically pretreated fibers showed more favorable structure than nanofibers separated from pulp fibers by the strong acid hydrolysis method (Siró and Plackett 2010). Even after the combined enzymatic and mechanical treatment, the NFC could still preserve the fiber 
length and have large length-diameter ratio (Wang et al. 2018). This property indicates that NFC could be used as a good reinforcement phase to fabricate biomaterials.

NFC is considered to be one of the most promising biomaterials in the polymer composites area in recent years (Kanmani et al. 2017; Agustin et al. 2018). It has the advantage that it can be produced and stored in the form of stable suspensions in water. The abundant hydroxyl groups are beneficial for further chemical modification and biomaterial fabrication. Many studies have shown that the NFC could reinforce the mechanical properties of polymer composites, such as NFC/poly(e-caprolactone) (PCL) paper, NFC/polyaniline (PANI) nano-composite, NFC/polyethylene (PE) composite, NFC/polyhydroxybutyrate (PHB) films, NFC/graphene composite films, and so on (Siró and Plackett 2010; Yadav et al. 2013; Dang and Seppälä 2015; El Miri et al. 2016). The main forming method of NFC-based films are the casting method and the evaporation method. The casting method is a process of components self-assembly process under the certain condition of vacuum, while evaporation method is a self-assembly process at normal atmospheric pressure. It is worth emphasizing that the NFC-based films fabricated by different forming methods could lead to different structure and properties. Moreover, for the application of the NFC-based composites, especially in the high-temperature insulation and fire-retardant materials area, the thermostability property is a very important performance parameter (Wicklein et al. 2014). However, the thermal pyrolysis process and mechanism of NFC composites have not been extensively studied.

Graphene oxide (GO) has attracted significant interest around the world owing to its outstanding physical properties and wide range of possible modifications (Dang and Seppälä 2015; Wan and Li 2016). GO is usually produced via exfoliating graphite oxide, which is synthesized by oxidizing graphite based on Hummers' method (Yadav et al. 2013). GO has two advantages: its hydrophilic characteristic and abundant oxygen functionalities. Due to these properties, GO can be dispersed in water and form stable GO suspensions. Therefore, GO can be compatible with many hydrophilic polymer matrices, such as poly(vinyl alcohol), plant fibers, cellulose, sodium carboxymethyl cellulose, and nanocrystalline cellulose acetate (Siró and Plackett 2010; Yadav et al. 2013; Kabiri and Namazi 2014). Moreover, fabrication of graphene-based products with bio-degradable and renewable polymers are important research topics in the renewable materials field. Therefore, NFC/GO composites may have a potential application in the high-temperature insulation and fire-retardant materials area. The thermal behaviors of NFC/GO composites play an important role in its applications. Generally, the thermal pyrolysis mechanism of biomass or materials is analyzed by using a thermogravimetric analyzer based on a lot of pyrolysis models, such as the Coats-Redfern model, Kissinger-Akahira-Sunose (KAS) model, Flynn-Wall-Ozawa (FWO) model, Mater-plots method, and Free Friedman model (Chen et al. 2012; Gao et al. 2017). In these models, Coats-Redfern model, FWO model, and KAS model are widely used because of their good reliability. The thermal decomposition of NFC is a complex process, and it may have different reactions simultaneously, e.g. dehydration, fragmentation, re-arrangement, re-polymerization, and carbonization (Lei et al. 2020). Acid, aldehydes, ketone, sugar anhydride, and gases (CO, $\mathrm{CO}_{2}$, and $\mathrm{CH}_{4}$ ) will also be generated in the thermal pyrolysis (Werner et al. 2014). However, the GO have totally different structure and chemical components compared with $\mathrm{NFC}$, and the pyrolysis mechanism of NFC/GO composites is not clearly known. Investigation the pyrolysis of these composites will promote the application of NFC/GO at high temperatures. 
In this study, nanofibrillated cellulose (NFC) and graphene oxide (GO) were prepared by enzymatic pretreatment/mechanical nano-fibrillation method and modified Hummers method, respectively. The NFC/GO composites were fabricated by casting method and evaporation method. The relationship between the microstructure and thermal decomposition properties of NFC/GO composites fabricated by two different methods were investigated using SEM and thermogravimetric analysis. The thermal pyrolysis mechanism of NFC/GO composites was carried out by thermogravimetric analyzer (TGA) and analyzed by Coats-Redfern model based on the TG data. This research may provide an important basis of theory for the fabrication and application of NFC/GO composites.

\section{EXPERIMENTAL}

\section{Materials}

Bleached eucalyptus pulps (BEP) were obtained from Zhejiang Yongtai Paper Group Co., Ltd (Hangzhou, China). The chemical composition of BEP was $78.1 \%$ glucan, $15.0 \%$ xylan, and $0.7 \%$ Klason lignin (Xiang et al. 2016). Cellulases (C2730, with an activity of approximately $220 \mathrm{FPU} / \mathrm{mL}$ ) and graphite microcrystalline (powder, $300 \mathrm{mesh}$ ) were purchased from Sigma. All other chemicals used in this study were reagent grade.

\section{Preparation of nanofibrillated cellulose}

The nanofibrillated cellulose (NFC) was prepared by an enzymatic hydrolysis pretreatment/mechanical nano-fibrillation method from BEP. Enzymatic hydrolysis pretreatment of BEP $(250.0 \mathrm{~g})$ was carried out at solids loading of $2.0 \%(\mathrm{w} / \mathrm{v})$ in a sodium acetate buffer of $\mathrm{pH} 4.8$ with a cellulase loading of $3 \mathrm{FPU} / \mathrm{g}$ substrate. The slurry was placed in an $18 \mathrm{~L}$ reactor at $50{ }^{\circ} \mathrm{C}$ with $200 \mathrm{rpm}$ for $6 \mathrm{~h}$. Then, the slurry was separated, and enzymatic hydrolysis residues were disintegrated in a blender at $20,000 \mathrm{rpm}$ for $30 \mathrm{~s}$ to make a $2.0 \mathrm{wt} \%$ suspension. The suspension was further diluted to $0.2 \mathrm{wt} \%$ and passed through a micro-fluidizer (M-110 EHIS, Newton, MA, USA) with 20 passes in a $200 \mu \mathrm{m}$ chamber and 20 additional passes in an $87 \mu \mathrm{m}$ chamber. The diameter distribution of obtained NFC was mainly distributed in the range 20 to $40 \mathrm{~nm}$, based on an analysis by field emission scanning electron microscope (FE-SEM) (Merlin, Carl Zeiss, Germany), as shown in Fig. 1.
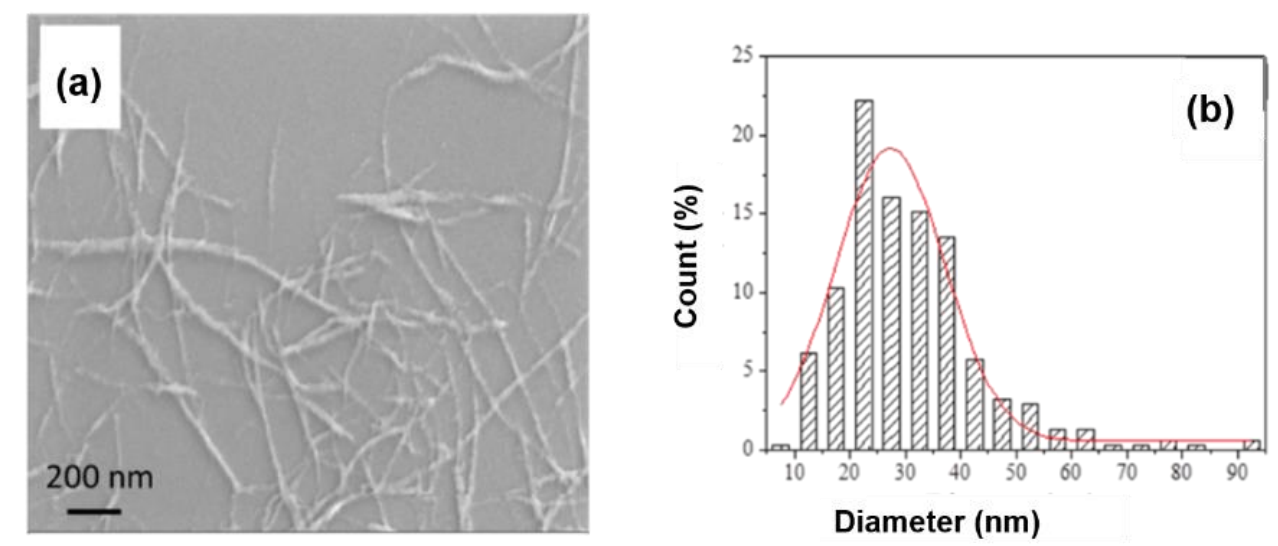

Fig. 1. The SEM analysis: (a) the morphological structure of cellulose nanofibrils (NFC); (b) the diameter distribution of NFC 


\section{Preparation of graphene oxide}

Graphene oxide (GO) was prepared with the modified Hummers method (Yang et al. 2010). Briefly, graphite powder (3 g), $\mathrm{P}_{2} \mathrm{O}_{5}(2.5 \mathrm{~g})$, and $\mathrm{K}_{2} \mathrm{~S}_{2} \mathrm{O}_{8}(2.5 \mathrm{~g})$ were firstly mixed in $96 \mathrm{wt} \% \mathrm{H}_{2} \mathrm{SO}_{4}(12 \mathrm{~mL})$, and this mixture were reacted at $80{ }^{\circ} \mathrm{C}$ for $12 \mathrm{~h}$. Then, the mixture was separated by centrifugation, and here the solid was called pre-oxidized graphite. After washing and drying, the pre-oxidized graphite was added into $96 \%$ sulfuric acid $(120 \mathrm{~mL})$ on an ice bath. Next, $\mathrm{KMnO}_{4}(15 \mathrm{~g})$ was gradually added into the mixture. After that, the mixture was kept at $35^{\circ} \mathrm{C}$ for $2 \mathrm{~h}$ and diluted with $200 \mathrm{~mL}$ ice water (keep the mixture temperature below $40{ }^{\circ} \mathrm{C}$ ). This diluted mixture was kept stirred at $25^{\circ} \mathrm{C}$ for 2 h. Then, deionized water $(400 \mathrm{~mL})$ and $30 \% \mathrm{H}_{2} \mathrm{O}_{2}(50 \mathrm{~mL})$ were added into the mixture to stop the oxidization reaction. The light yellow product was filtered and washed several times, then dried for analysis. As shown in Fig. 2, the FT-IR (Tensor 27/Hyperion) spectrogram of $\mathrm{GO}$ showed that $\mathrm{C}=\mathrm{C}=\mathrm{C}, \mathrm{C}=\mathrm{O}, \mathrm{C}=\mathrm{C}, \mathrm{C}-\mathrm{O}-\mathrm{C}$, and $\mathrm{C}-\mathrm{O}-\mathrm{H}$ groups appeared at $2361 \mathrm{~cm}^{-1}, 1722 \mathrm{~cm}^{-1}, 1624 \mathrm{~cm}^{-1}, 1264 \mathrm{~cm}^{-1}$, and $1400 \mathrm{~cm}^{-1}$, respectively, which indicated that the graphite was totally oxidized to GO. The atomic force microscope (AFM, Multimode 8, Bruker, Germany) analysis (Fig. 3) of GO showed that graphite oxide was successfully exfoliated to GO, and the thickness of GO was about 0.3 to $0.7 \mathrm{~nm}$ (1 to 3 layers). One single layer thickness of $\mathrm{GO}$ is about $0.3 \mathrm{~nm}$.

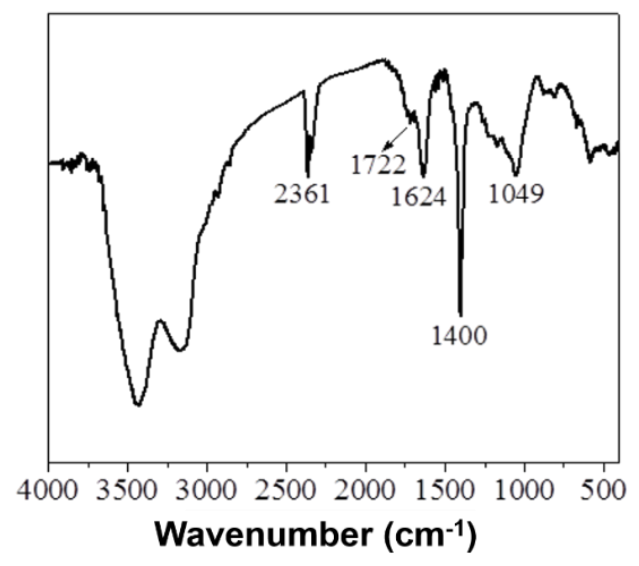

Fig. 2. The FT-IR analysis of graphene oxide (GO)
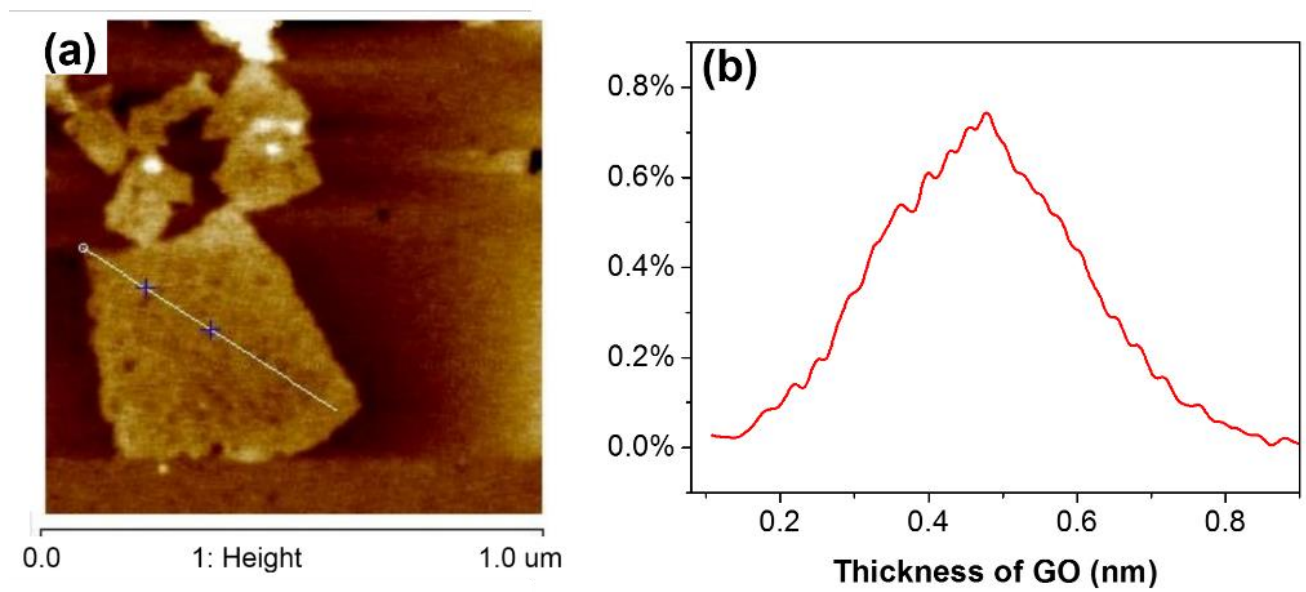

Fig. 3. The AFM analysis: (a) the morphology of graphene oxide (GO); (b) the thickness of GO 
Preparation of cellulose nanofibrils/graphene oxide composites

The graphene oxide (GO) was prepared for water solution at $1 \mathrm{mg} / \mathrm{mL}$ by using an ultra-sonication bath to yield a stable brown solution. The NFC/GO biocomposite was manufactured following the procedure described by Valentini et al. (2013). The nanofibrillated cellulose (NFC) was added to the GO suspension with the ratio of NFC and GO (weight) 1:1. The mixing was performed in an ultra-sonication bath for $30 \mathrm{~min}$ at 25 ${ }^{\circ} \mathrm{C}$. Then, the mixture was molded by the casting method and the evaporation method, and the products were labeled for NFC/GO-C composites and NFC/GO-E composites respectively. After that, all of the samples were cleaned several times with acetone and dried in vacuum at $100{ }^{\circ} \mathrm{C}$ for $1 \mathrm{~h}$.

The morphological structure of cellulose nanofibrils/graphene oxide composites

A field emission scanning electron microscope (FE-SEM) (Merlin, Carl Zeiss, Germany) was used to analyze the longitudinal microstructure of NFC/GO composites.

\section{Thermogravimetric analysis}

The thermogravimetric analysis (TGA) of NFC/GO film was carried out with a thermogravimetric analyzer (TG Q500, TG Cor. USA). The instrument could record the sample weight-losses with changes of temperature. For each test, about $10 \mathrm{mg}$ of sample was placed in an aluminum pan of the thermal analyzer. The sizing of testing sample was about $5 \times 5 \mathrm{~mm}$. In the testing process, the high purity nitrogen flow rate was used as carried gas with the flow rate of $25 \mathrm{~mL} / \mathrm{min}$. The temperature rose from ambient temperature to $800{ }^{\circ} \mathrm{C}$ with the heating rate of $10{ }^{\circ} \mathrm{C} / \mathrm{min}$. All samples were heated to $50{ }^{\circ} \mathrm{C}$ and held 15 min until obtaining a constant mass. Each experiment was repeated at least three times.

\section{Kinetic model}

The kinetic equation of heterogeneous solid reactions under non-isothermal conditions can be summarized by Eq. (1) (Ceylan et al. 2014; Ali et al. 2017; Gao and Chen 2017),

$$
\frac{d \alpha}{d t}=k(T) f(\alpha)
$$

where $\alpha$ and $T$ are the conversion and absolute temperature, and $\alpha$ is defined as in Eq. 2,

$$
\alpha=\frac{M_{0}-M_{t}}{M_{0}-M_{\infty}}
$$

where $M_{0}, M_{\mathrm{t}}$, and $M_{\infty}$ refer to initial, instantaneous, and final weights, respectively. The parameter $k(T)$ is the temperature-dependent reaction rate constant, as follows:

$$
k(T)=A \exp \left(\frac{-E}{R T}\right)
$$

In Eq. 3, $A\left(\mathrm{~s}^{-1}\right)$ is the pre-exponential or frequency factor; $E(\mathrm{~J} / \mathrm{mol})$ is the activation energy; and $R$ is the universal gas constant. Substituting Eq. 3 into Eq. 1 obtains,

$$
\frac{d \alpha}{d t}=A \exp \left(\frac{-E}{R T}\right) f(\alpha)
$$

where $f(\alpha)$ represents the reaction model which is a function of $\alpha$, which is usually expressed as follows:

$$
f(\alpha)=(1-\alpha)^{n}
$$


Table 1. Most Frequently Used Thermal Mechanisms of Solid-State Degradation Processes (Chen et al. 2012)

\begin{tabular}{|c|c|c|c|}
\hline Mechanisms & Symbol & $f(\alpha)$ & $g(\alpha)$ \\
\hline \multicolumn{4}{|l|}{ Order of reaction } \\
\hline First-order & $\mathrm{F} 1$ & $1-\alpha$ & $-\ln (1-\alpha)$ \\
\hline Second-order & $\mathrm{F} 2$ & $(1-\alpha)^{2}$ & $(1-\alpha)^{-1}-1$ \\
\hline Third-order & F3 & $(1-\alpha)^{3}$ & {$\left[(1-\alpha)^{-2}-1\right] / 2$} \\
\hline \multicolumn{4}{|l|}{ Diffusion } \\
\hline One-way transport & $\mathrm{D} 1$ & $0.5 \alpha$ & $\alpha^{2}$ \\
\hline Two-way transport & D2 & {$[-\ln (1-\alpha)]^{-1}$} & $\alpha+(1-\alpha) \ln (1-\alpha)$ \\
\hline Three-way transport & D3 & $1.5(1-\alpha)^{2 / 3}\left[1-(1-\alpha)^{1 / 3}\right]^{-1}$ & {$\left[1-(1-\alpha)^{1 / 3}\right]^{2}$} \\
\hline Ginstling-Brounstein equation & D4 & $1.5\left[(1-\alpha)^{1 / 3}-1\right]$ & $(1-2 \alpha / 3)-(1-\alpha)^{2 / 3}$ \\
\hline \multicolumn{4}{|c|}{$\begin{array}{l}\text { Limiting surface reaction between both } \\
\text { phases }\end{array}$} \\
\hline One dimension & $\mathrm{R} 1$ & $\overline{1}$ & $\alpha$ \\
\hline Two dimensions & $\mathrm{R} 2$ & $2(1-\alpha)^{1 / 2}$ & $1-(1-\alpha)^{1 / 2}$ \\
\hline Three dimensions & R3 & $3(1-\alpha)^{2 / 3}$ & $1-(1-\alpha)^{1 / 3}$ \\
\hline \multicolumn{4}{|c|}{ Random nucleation and nuclei growth } \\
\hline Two-dimensional & A2 & $2(1-\alpha)[-\ln (1-\alpha)]^{1 / 2}$ & {$[-\ln (1-\alpha)]^{1 / 2}$} \\
\hline Three-dimensional & A3 & $3(1-\alpha)[-\ln (1-\alpha)]^{2 / 3}$ & {$[-\ln (1-\alpha)]^{1 / 3}$} \\
\hline \multicolumn{4}{|l|}{ Exponential nucleation } \\
\hline Power law, $n=1 / 2$ & P2 & $2 \alpha^{1 / 2}$ & $\alpha^{1 / 2}$ \\
\hline Power law, $n=1 / 3$ & P3 & $3 a^{2 / 3}$ & $\alpha^{1 / 3}$ \\
\hline Power law, $n=1 / 4$ & $\mathrm{P} 4$ & $4 \alpha^{3 / 4}$ & $a^{1 / 4}$ \\
\hline
\end{tabular}

The heating rate is defined as $\beta$ (Gao et al. 2012),

$$
\beta=\frac{d T}{d t}
$$


which gives,

$$
\frac{d \alpha}{d T}=\frac{A}{\beta} \exp \left(\frac{-E}{R T}\right)(1-\alpha)^{n}
$$

The Coats-Redfern model is the usual model employed to investigate the thermal decomposition of solid. Combining Eqs. 2 and 6, the Coats-Redfern equation can be outlined as follows (Gao et al. 2012, 2014; Ceylan et al. 2014):

$$
\frac{d \alpha}{f(\alpha)}=\frac{A}{\beta} \exp \left(\frac{-E}{R T}\right) d T
$$

An integral function was defined:

$$
\mathrm{g}(\alpha)=\frac{A}{\beta} \int_{T_{0}}^{T} \exp \left(-\frac{E}{R T}\right) d T
$$

where, $T_{0}$ is the initial temperature.

Here, the Coats-Redfern equation is derived as follows:

$$
\ln \left[\frac{\mathrm{g}(\alpha)}{T^{2}}\right]=\ln \left[\frac{A R}{\beta E}\left(1-\frac{2 R T}{E}\right)\right]-\frac{E}{R T}
$$

Due to the fact that $\frac{2 R T}{E} \ll 1$, in Eq. (10), $\ln \left[\frac{\mathrm{g}(\alpha)}{T^{2}}\right]$ and $\left(\frac{1}{T}\right)$ has a linear relationship, and $E$ and $A$ can be calculated through fitting the line. The most frequently used reaction models of $f(\alpha)$ and $g(\alpha)$ are listed in Table 1 and based on Eq. 10 the values of activation energy $(E)$ and frequency factors $(A)$ can be calculated.

\section{RESULTS AND DISCUSSION}

\section{Microstructure of NFC/GO Composites}

Longitudinal section microstructures of NFC/GO composites made by casting method and evaporation method were analyzed by SEM (Fig. 4). Figure 4 shows that the $\mathrm{NFC/GO}$ composites had relatively uniform layered microstructure. Compared to the NFC/GO-E composites, NFC/GO-C composites had more evenly distributed structure. The NFC/GO-E composites had some cracks, as shown in Fig. 4. The different microstructural features between NFC/GO-E composites and NFC/GO-C composites may affect the uniformity of bonding of NFC and GO, resulting in different physical properties of the composites.
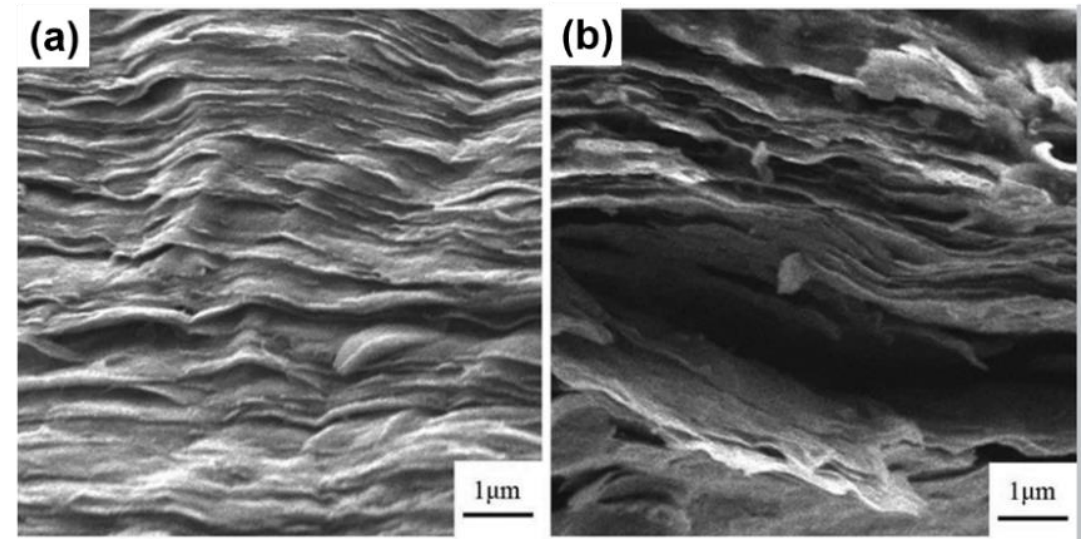

Fig. 4. Longitudinal section microstructures of NFC/GO-C composites (a) and NFC/GO-E composites (b) 


\section{Thermal Pyrolysis Properties of NFC/GO Composites}

Figure 5 shows the TG and DTG curves of the NFC/GO-C composites and NFC/GO-E composites. When the thermogravimetric analysis was carried out to $800{ }^{\circ} \mathrm{C}$, the final weights were about 32.0 to $34.0 \%$ for the two kinds of samples (Fig. 5a). The weight-loss was mainly from the thermal decomposition of NFC and GO, especially the cellulose/hemicellulose of NFC and the oxygen-containing groups of $\mathrm{GO}$, e.g. $\mathrm{C}=\mathrm{O}, \mathrm{C}-\mathrm{O}-$ $\mathrm{C}$, and C-O-H (Shen and Gu 2009). Additionally, as can be seen from Fig. 5a and b, above $500{ }^{\circ} \mathrm{C}$ weight-loss peak was hardly apparent due to the further devolatilization of the formed biochar gradually, and the breakdown of $\mathrm{C}-\mathrm{C}$ and $\mathrm{C}-\mathrm{H}$ bonds from the film (Gao et al. 2017). As shown in Fig.5b, the minor and major mass loss rate of NFC/GO-C composites occurred at $200{ }^{\circ} \mathrm{C}$ and $307{ }^{\circ} \mathrm{C}$, respectively. However, for NFC/GO-E composites the mass loss shoulder peaks appeared at $205{ }^{\circ} \mathrm{C}$ and $241{ }^{\circ} \mathrm{C}$, respectively. Therefore, the thermal degradation of these NFC/GO composites could be divided into two processes, which could be ascribed to the main decomposition stage of 100 to $220{ }^{\circ} \mathrm{C}$ and 220 to $380{ }^{\circ} \mathrm{C}$.
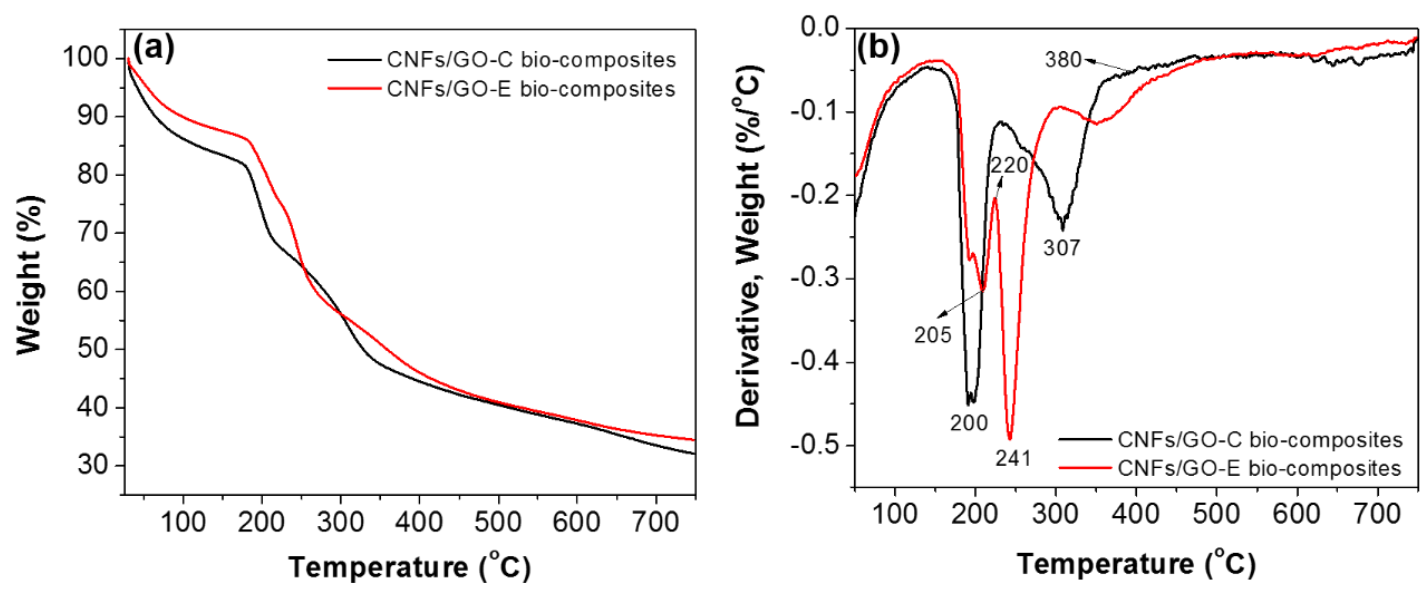

Fig. 5. Thermogravimetric curves (TG), (a) and differential thermogravimetric curves (DTG), (b) of NFC/GO composites

\section{Kinetic Analysis}

In order to analyze the thermal pyrolysis parameters of the composites, the CoatsRedfern model was introduced. The two main thermal decomposition stages of 100 to 220 ${ }^{\circ} \mathrm{C}$ and 220 to $380{ }^{\circ} \mathrm{C}$ were considered, respectively. By calculating the weight-loss rate $\alpha$ from the curves in Fig. 4 and using the reaction mechanism $g(\alpha)$ from Table 1, fitting curves and coefficients of determination $\left(\mathrm{R}^{2}\right)$ with different reaction models of NFC/GO composites were obtained (Figs. 6 and 7, Tables 2 and 3) based on the TG and DTG data in the two main thermal decomposition stages. 
(a)

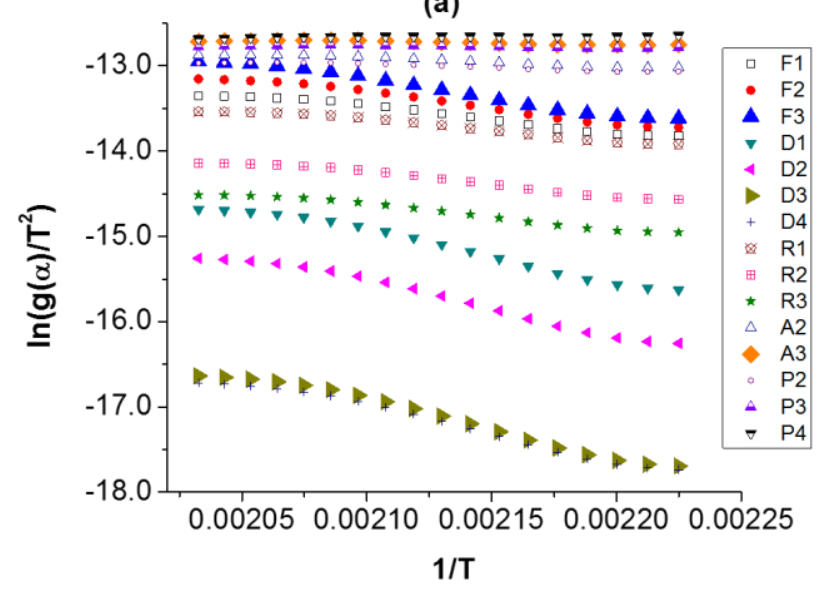

(b)

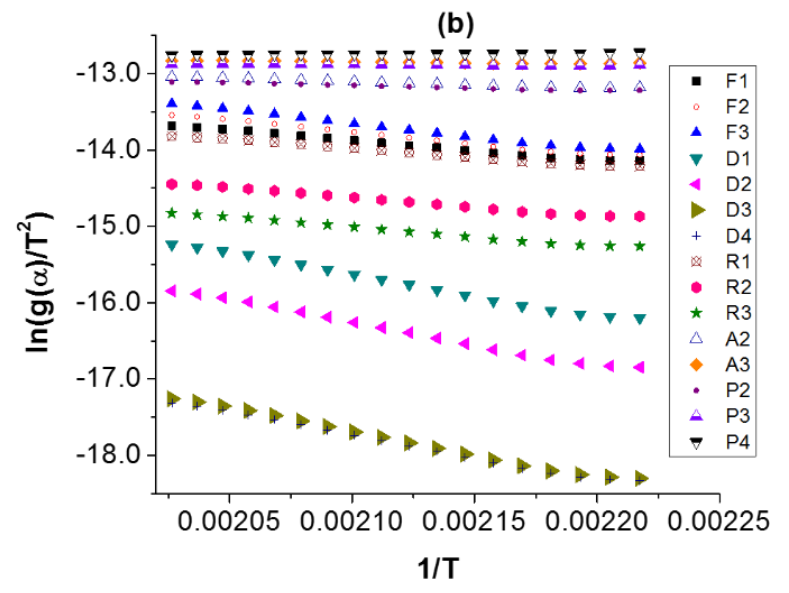

Fig. 6. Coats-Redfern fitting plots of NFC/GO-C composites (a) and NFC/GO-E composites (b) at the temperature stage of $100 \sim 220^{\circ} \mathrm{C}$

Within the thermal pyrolysis stage of 100 to $220^{\circ} \mathrm{C}$, all of the reaction mechanisms in Table 1 except mechanisms of $\mathrm{A} 3, \mathrm{P} 3$, and $\mathrm{P} 4$ could be used to describe the thermal decomposition mechanism of NFC/GO-C composites (Table 2), which was corresponded by fitting curves with $\mathrm{R}^{2}$ more than 0.9 (Fig. 6, Table 2). The diffusion model D4 (Ginstling-Brounstein equation) had the highest $\mathrm{R}^{2}$ of 0.9796 with an activation energy $(E)$ value of $50.4 \mathrm{~kJ} / \mathrm{mol}$ (Table 2), and considered as the most possible reaction model in this temperature stage. However, for the NFC/GO-E composites, diffusion model D1 had the highest $\mathrm{R}^{2}$ of 0.9952 with activation energy $(E)$ value of $46.3 \mathrm{~kJ} / \mathrm{mol}$ (Table 2). Power law P3 and P4 models were also not suitable for describing the thermal pyrolysis process of film (Table 2). In this temperature stage, the weight-loss was mainly attributed to the bonding breaking between NFC and GO, such as the dehydration of carboxylic group, carbonyl group, hydrogen bond, etc. (Liu et al. 2010).

The carbohydrate from NFC consisted of arabinan, galactan, glucan, and xylan, which could also begin to decompose in this stage (Ceylan et al. 2014; Gao and Chen 2017; Gao et al. 2017). Therefore, in this temperature stage the two kinds of NFC/GO composites had the similar thermal mechanisms although they were mold by casting method or evaporation method. 
Table 2. Fitted Equations, Coefficients of Determination $\left(\mathrm{R}^{2}\right)$, and Activation Energies $(E, \mathrm{~kJ} / \mathrm{mol})$ Obtained from the Coats-Redfern Model within the Temperature Range of 100 to $220^{\circ} \mathrm{C}$

\begin{tabular}{|c|c|c|c|c|c|c|}
\hline \multirow{2}{*}{ Mechanisms } & \multicolumn{3}{|c|}{ NFC/GO-C composites } & \multicolumn{3}{|c|}{ NFC/GO-E composites } \\
\hline & Fitted equation & $R^{2}$ & $E$ & Fitted equation & $\mathrm{R}^{2}$ & $E$ \\
\hline $\mathrm{F} 1$ & $y=-2848.79 x-7.51$ & 0.9745 & 23.68 & $y=-2665.09 x-8.28$ & 0.9930 & 22.16 \\
\hline $\mathrm{F} 2$ & $y=-3416.72 x-6.15$ & 0.9789 & 28.41 & $y=-3042.13 x-7.37$ & 0.9926 & 25.29 \\
\hline F3 & $y=-4068.19 x-4.63$ & 0.9835 & 33.82 & $y=-3444.15 x-6.42$ & 0.9921 & 28.64 \\
\hline D1 & $y=-5609.22 x-3.18$ & 0.9781 & 46.64 & $y=-5569.69 x-3.93$ & 0.9952 & 46.31 \\
\hline D2 & $y=-5935.38 x-3.09$ & 0.9793 & 49.35 & $y=-5796.08 x-4.08$ & 0.9951 & 48.19 \\
\hline D3 & $y=-6284.28 x-3.76$ & 0.9804 & 52.25 & $y=-6033.38 x-5.02$ & 0.9949 & 50.16 \\
\hline D4 & $y=-6061.58 x-4.32$ & 0.9796 & 50.40 & $y=-5875.14 x-5.40$ & 0.9950 & 48.85 \\
\hline $\mathrm{R} 1$ & $y=-2333.70 x-8.75$ & 0.9680 & 19.40 & $y=-2313.14 x-9.12$ & 0.9932 & 19.23 \\
\hline $\mathrm{R} 2$ & $y=-2584.65 x-8.84$ & 0.9716 & 21.49 & $y=-2485.98 x-9.40$ & 0.9931 & 20.67 \\
\hline R3 & $y=-2671.23 x-9.03$ & 0.9726 & 22.21 & $y=-2544.99 x-9.66$ & 0.9930 & 21.16 \\
\hline $\mathrm{A} 2$ & $y=-953.49 x-10.91$ & 0.9431 & 7.93 & $y=-860.85 x-11.30$ & 0.9843 & 7.16 \\
\hline A3 & - & - & - & $y=-259.43 x-12.30$ & 0.9307 & 2.16 \\
\hline $\mathrm{P} 2$ & $y=-695.94-11.53$ & 0.9121 & 5.79 & $y=-684.87 x-11.72$ & 0.9816 & 5.69 \\
\hline P3/P4 & - & - & - & - & - & - \\
\hline
\end{tabular}

Note: - represents $R^{2}$ is less than 0.9 , and the reaction mechanism is not suitable.

At the temperature stage of 220 to $380{ }^{\circ} \mathrm{C}$, the different forming methods of NFC/GO composites resulted in different thermal pyrolysis mechanisms (Fig. 7, Table 3). For the NFC/GO-E composites, only $\mathrm{P} 3$ and $\mathrm{P} 4$ reaction equations could exactly reflect the thermal pyrolysis process. However, for the NFC/GO-C composites, except limiting surface reactions R1, R2, and R3, all of the other reactions could be suitable for analysis the thermal decomposition mechanism. The reason may be that this temperature stage was the main decomposition stage of hemicellulose, whose thermal pyrolysis temperature was 200 to $280{ }^{\circ} \mathrm{C}$ (Liu et al. 2010). This result indicated that the forming method obviously affected the thermal pyrolysis mechanism of composites in the 220 to $380{ }^{\circ} \mathrm{C}$ temperature stage. The uniform layered microstructure of NFC/GO-C composites (Fig. 4) led to a relatively consonant thermal decomposition process, which could be described by one thermal mechanism. 

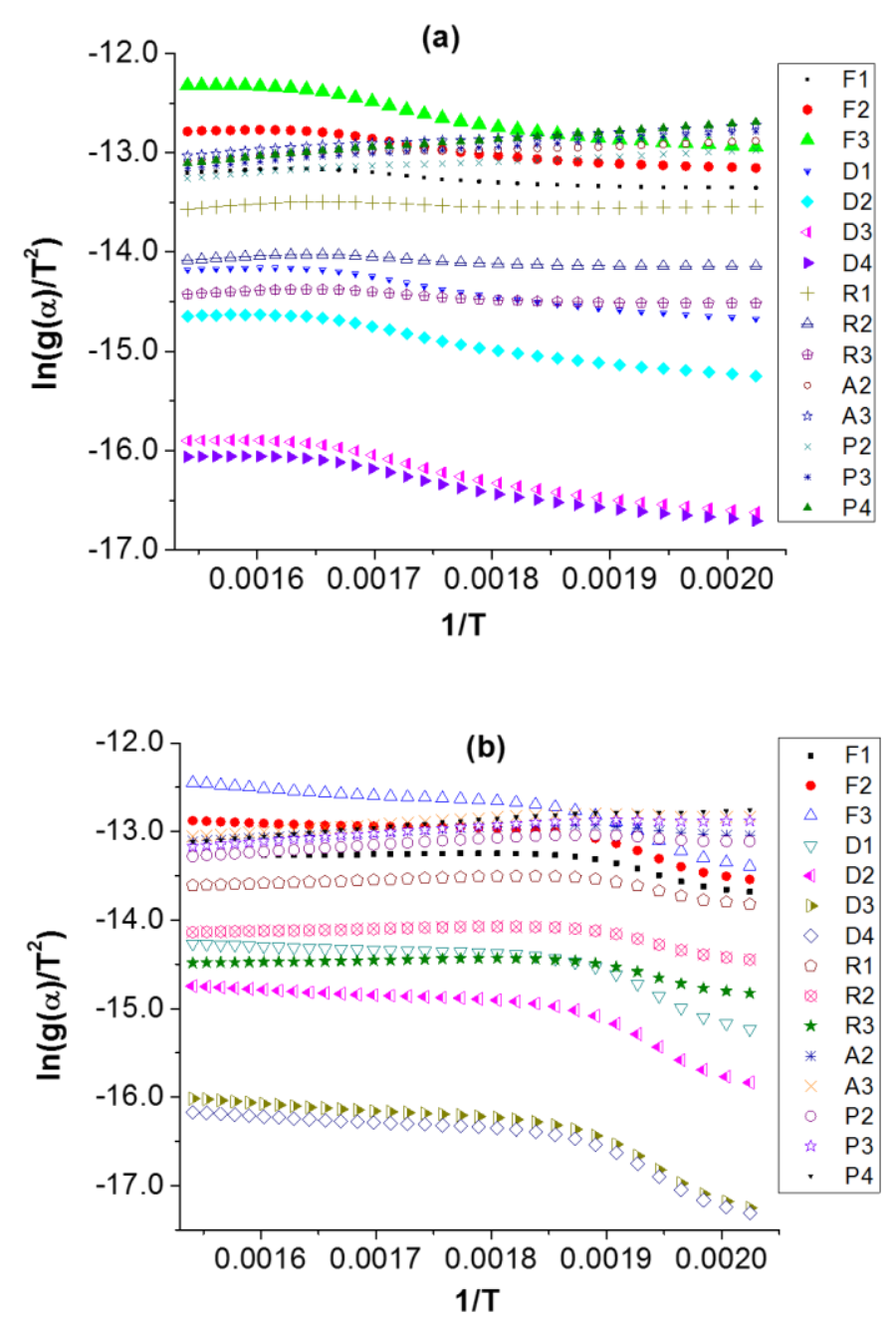

Fig. 7. Coats-Redfern fitting plots of NFC/GO-C composites (a) and NFC/GO-E composites (b) at the temperature stage of $220 \sim 380^{\circ} \mathrm{C}$ 
Table 3. Fitted Equations, Coefficients of Determination $\left(R^{2}\right)$ and Activation Energies $(E, \mathrm{~kJ} / \mathrm{mol})$ Obtained by Coats-Redfern Model within the Temperature Range of 220 to $380^{\circ} \mathrm{C}$

\begin{tabular}{|c|c|c|c|c|c|c|}
\hline \multirow{2}{*}{ Mechanisms } & \multicolumn{3}{|c|}{ NFC/GO-C composites } & \multicolumn{3}{|c|}{ NFC/GO-E composites } \\
\hline & Fitted equation & $\mathrm{R}^{2}$ & $E$ & Fitted equation & $\mathrm{R}^{2}$ & $E$ \\
\hline $\mathrm{F} 1$ & $Y=-479.32 x-12.41$ & 0.9093 & 3.99 & - & - & - \\
\hline $\mathrm{F} 2$ & $Y=-985.77 x-11.21$ & 0.9459 & 8.20 & - & - & - \\
\hline F3 & $Y=-1578.87 x-9.83$ & 0.9614 & 13.13 & - & - & - \\
\hline $\mathrm{D} 1$ & $Y=-1260.92 x-12.15$ & 0.9571 & 10.48 & - & - & - \\
\hline $\mathrm{D} 2$ & $Y=-1510.03 x-12.23$ & 0.9644 & 12.56 & - & - & - \\
\hline D3 & $Y=-1796.48 x-13.04$ & 0.9695 & 14.94 & - & - & - \\
\hline D4 & $Y=-1605.16 x-13.51$ & 0.9664 & 13.35 & - & - & - \\
\hline $\mathrm{R} 1 / \mathrm{R} 2 / \mathrm{R} 3$ & - & - & - & - & - & - \\
\hline $\mathrm{A} 2$ & $Y=328.08 x-13.55$ & 0.9240 & 2.73 & - & - & - \\
\hline A3 & $Y=597.22 x-13.93$ & 0.9869 & 4.97 & - & - & - \\
\hline P2 & $Y=536.38 x-14.06$ & 0.9774 & 4.46 & - & - & - \\
\hline P3 & $Y=736.08 x-14.27$ & 0.9925 & 6.12 & $Y=686.94 x-14.20$ & 0.9250 & 5.71 \\
\hline P4 & $Y=835.93 x-14.37$ & 0.9955 & 6.95 & $Y=799.07 x-14.32$ & 0.9632 & 6.64 \\
\hline
\end{tabular}

Note: - represents $R^{2}$ is less than 0.9 , and the reaction mechanism is not suitable.

\section{CONCLUSIONS}

1. The microstructure and thermal pyrolysis mechanism of NFC/GO composites fabricated by casting method or evaporation method were investigated. NFC/GO-C composites had more uniform layered microstructure than NFC/GO-E composites, based on analysis using SEM.

2. For the thermal pyrolysis mechanism, in the temperature range of 100 to $220{ }^{\circ} \mathrm{C}$ the two kinds of composites showed similar thermal pyrolysis mechanisms, and diffusion mechanisms were considered as the most possible reaction model. However, in the stage of 220 to $380{ }^{\circ} \mathrm{C}$, the NFC/GO composites had different thermal pyrolysis mechanism equations due to the forming method differently. Only P3 and P4 reaction equations could exactly reflect the thermal pyrolysis process of NFC/GO-E composites. 
3. The forming method could affect the properties of NFC/GO composites, especially the microstructure and thermostability. This study may support for the fabrication and application of NFC/GO-based materials.

\section{ACKNOWLEDGMENTS}

The authors are grateful for the support the Foundation (No.KF201802) of State Key Laboratory of Biobased Material and Green Papermaking, Qilu University of Technology, Shandong Academy of Sciences, National Natural Science Foundation of China (31971603), and Fundamental Research Funds for the Central Universities (2019MS084).

\section{REFERENCES CITED}

Agustin, M. B., Nakatsubo, F., and Yano, H. (2018). "Improving the thermal stability of wood-based cellulose by esterification," Carbohydrate Polymers 192, 28-36. DOI: 10.1016/j.carbpol.2018.02.071

Alemdar, A., and Sain, M. (2008). "Isolation and characterization of nanofibers from agricultural residues - Wheat straw and soy hulls," Bioresource Technology 99, 1664-1671. DOI: 10.1016/j.biortech.2007.04.029

Ali, I., Bahaitham, H., and Naebulharam, R. (2017). "A comprehensive kinetics study of coconut shell waste pyrolysis," Bioresource Technology 235, 1-11. DOI: 10.1016/j.biortech.2017.03.089

Beltramino, F., Roncero, M. B., Vidal, T., Torres, A.L., and Valls, C. (2015). "Increasing yield of nanocrystalline cellulose preparation process by a cellulase pretreatment," Bioresource Technology 192, 574-581. DOI: 10.1016/j.biortech.2015.06.007

Ceylan, S., Topcu, Y., and Ceylan, Z. (2014). "Thermal behaviour and kinetics of alga Polysiphonia elongata biomass during pyrolysis," Bioresource Technology 171, 193198. DOI: 10.1016/j.biortech.2014.08.064

Chen, C., Ma, X., and He, Y. (2012). "Co-pyrolysis characteristics of microalgae Chlorella vulgaris and coal through TGA," Bioresource Technology 117, 264-273. DOI: 10.1016/j.biortech.2012.04.077

Dang, L. N., and Seppälä, J. (2015). "Electrically conductive nanocellulose/graphene composites exhibiting improved mechanical properties in high-moisture condition," Cellulose 22, 1799-1812. DOI: 10.1007/s10570-015-0622-2

El Miri, N., El Achaby, M., Fihri, A., Larzek, M., Zahouily, M., Abdelouahdi, K., Barakat, A., and Solhy, A. (2016). "Synergistic effect of cellulose nanocrystals/ graphene oxide nanosheets as functional hybrid nanofiller for enhancing properties of PVA nanocomposites," Carbohydrate Polymers 137, 239-248. DOI: 10.1016/j.carbpol.2015.10.072

Gao, W., and Chen, K. (2017). "Physical properties and thermal behavior of reconstituted tobacco sheet with precipitated calcium carbonate added in the coating process," Cellulose 24, 2581-2590. DOI: 10.1007/s10570-017-1270-5

Gao, W., Chen, K., Xiang, Z., Yang, F., Zeng, J., Li, J., Yang, R., Rao, G., and Tao, H. (2012). "Kinetic study on pyrolysis of tobacco residues from the cigarette industry," Industrial Crops and Products 44, 152-157. DOI: 10.1016/j.indcrop.2012.10.032 
Gao, W., Chen, K., Yang, R., and Yang, F. (2014). "Rheological property of reconstituted tobacco coatings," Industrial Crops and Products 60, 45-51. DOI: 10.1016/j.indcrop.2014.06.002

Gao, W., Chen, K., Zeng, J., Xu, J., and Wang, B. (2017). "Thermal pyrolysis characteristics of macroalgae Cladophora glomerate," Bioresource Technology 243, 212-217. DOI: 10.1016/j.biortech.2017.06.041

Kabiri, R., and Namazi, H. (2014). "Nanocrystalline cellulose acetate (NCCA)/graphene oxide (GO) nanocomposites with enhanced mechanical properties and barrier against water vapor," Cellulose 21, 3527-3539. DOI: 10.1007/s10570-014-0366-4

Kanmani, P., Aravind, J., Kamaraj, M., Sureshbabu, P., and Karthikeyan, S. (2017). "Environmental applications of chitosan and cellulosic biopolymers: A comprehensive outlook," Bioresource Technology 242, 295-303. DOI: 10.1016/j.biortech.2017.03.119

Lei, Z., Gao, W., Zeng, J., Wang, B., and Xu, J. (2020). “The mechanism of Cu (II) adsorption onto 2,3-dialdehyde nano-fibrillated celluloses," Carbohydrate Polymers 230, 115631. DOI: 10.1016/j.carbpol.2019.115631

Liu, H., Liu, D., Yao, F., and Wu, Q. (2010). "Fabrication and properties of transparent polymethylmethacrylate/cellulose nanocrystals composites," Bioresource Technology 101, 5685-5692. DOI: 10.1016/j.biortech.2010.02.045

Liu, W., Wang, B., Hou, Q., Chen, W., and Wu, M. (2016). "Effects of fibrillation on the wood fibers' enzymatic hydrolysis enhanced by mechanical refining," Bioresource Technology 206, 99-103. DOI: 10.1016/j.biortech.2016.01.074

Long, L., Tian, D., Hu, J., Wang, F., and Saddler, J. (2017). “A xylanase-aided enzymatic pretreatment facilitates cellulose nanofibrillation," Bioresource Technology 243, 898904. DOI: 10.1016/j.biortech.2017.07.037

Penttila, P. A., Varnai, A., Pere, J., Tammelin, T., Salmen, L., Siika-aho, M., Viikari, L., and Serimaa, R. (2013). "Xylan as limiting factor in enzymatic hydrolysis of nanocellulose," Bioresource Technology 129, 135-141. DOI: 10.1016/j.biortech.2012.11.017

Shen, D. K., and Gu, S. (2009). "The mechanism for thermal decomposition of cellulose and its main products," Bioresource Technology 100, 6496-6504. DOI: 10.1016/j.biortech.2009.06.095

Siró, I., and Plackett, D. (2010). "Microfibrillated cellulose and new nanocomposite materials: A review," Cellulose 17, 459-494. DOI: 10.1007/s10570-010-9405-y

Sun, S., Sun, S., Cao, X., and Sun, R. (2016). "The role of pretreatment in improving the enzymatic hydrolysis of lignocellulosic materials," Bioresource Technology 199, 4958. DOI: 10.1016/j.biortech.2015.08.061

Valentini, L., Cardinali, M., Fortunati, E., Torre, L., and Kenny, J. M. (2013). "A novel method to prepare conductive nanocrystalline cellulose/graphene oxide composite bio-composites," Materials Letters 105, 4-7. DOI: 10.1016/j.matlet.2013.04.034

Wan, C., and Li, J. (2016). "Graphene oxide/cellulose aerogels nanocomposite: Preparation, pyrolysis, and application for electromagnetic interference shielding," Carbohydrate Polymers 150, 172-179. DOI: 10.1016/j.carbpol.2016.05.051

Wang, S., Gao., Chen, K., Xiang, Z., Zeng, J., Wang, B., and Xu, J. (2018). "Deconstruction of cellulosic fibers to fibrils based on enzymatic pretreatment," Bioresource Technology 267, 426-430. DOI: 10.1016/j.biortech.2018.07.067 
Werner, K., Pommer, L., Broström, M. (2014). "Thermal decomposition of hemicelluloses," Journal of Analytical and Applied Pyrolysis 110, 130-137. DOI: 10.1016/j.jaap.2014.08.013

Wicklein, B., Kocjan, A., Salazar-Alvarez, G., Carosio, F., Camino, G., Antonietti, M., and Bergström, L. (2014). "Thermally insulating and fire-retardant lightweight anisotropic foams based on nanocellulose and graphene oxide," Nature Nanotechnology 10, 277-283. DOI: 10.1038/nnano.2014.248

Yadav, M., Rhee, K. Y., Jung, I. H., and Park, S. J. (2013). "Eco-friendly synthesis, characterization and properties of a sodium carboxymethyl cellulose/graphene oxide nanocomposite film," Cellulose 20, 687-698. DOI: 10.1007/s10570-012-9855-5

Yang, Q., Pan, X., Huang, F., and Li, K. (2010). "Fabrication of high-concentration and stable aqueous suspensions of graphene nanosheets by noncovalent functionalization with lignin and cellulose derivatives," Journal of Physical Chemistry C 114, 38113816. DOI: 10.1021/jp910232x

Xiang, Z., Gao, W., Chen, L., Lan, W., Zhu, J., and Runge, T. (2016). "A comparison of cellulose nanofibrils produced from Cladophora glomerata algae and bleached eucalyptus pulp," Cellulose 23, 493-503. DOI: 10.1007/s10570-015-0840-7

Article submitted: November 21, 2019; Peer review completed: March 8, 2020; Revised version received: April 30, 2020; Accepted: May 1, 2020; Published: May 8, 2020.

DOI: 10.15376/biores.15.3.4851-4865 